\title{
Scattering and Polarization Properties of the Scarab Beetle Cyphochilus insulanus cuticle
}

Christina Åkerlind, Hans Arwin, Tomas Hallberg, Jan Landin, Johan Gustafsson, Hans Kariis and Kenneth Järrendahl

\section{Linköping University Post Print}

\section{Tweet}

N.B.: When citing this work, cite the original article.

Original Publication:

Christina Åkerlind, Hans Arwin, Tomas Hallberg, Jan Landin, Johan Gustafsson, Hans Kariis and Kenneth Järrendahl, Scattering and Polarization Properties of the Scarab Beetle Cyphochilus insulanus cuticle, 2015, Applied Optics, (54), 19.

http://dx.doi.org/10.1364/A0.54.006037

Copyright: Optical Society of America http://www.osa.org/

Postprint available at: Linköping University Electronic Press http://urn.kb.se/resolve?urn=urn:nbn:se:liu:diva-115368 


\title{
Scattering and Polarization Properties of the Scarab Beetle Cyphochilus insulanus Cuticle
}

\author{
Christina Åkerlind,,${ }^{1,2^{*}}$ Hans Arwin, ${ }^{2}$ Tomas Hallberg, ${ }^{1}$ Jan Landin, ${ }^{2}$ Johan Gustafsson, ${ }^{3}$ Hans Kariis, ${ }^{1}$ \\ and Kenneth Järrendahl ${ }^{2}$ \\ ${ }^{1}$ Division of Sensor and Electronic Warfare, Swedish Defence Research Agency (FOI), SE 58111 Linköping, Sweden \\ ${ }^{2}$ Department of Physics, Chemistry and Biology, Linköping University, SE 58183 Linköping, Sweden \\ ${ }^{3}$ Division of Defence and Security, Systems and Technology, Swedish Defence Research Agency (FOI), \\ SE 14725 Tumba, Sweden \\ *Corresponding author: christina.akerlind@foi.se
}

Received Month X, XXXX; revised Month X, XXXX; accepted Month X, XXXX; posted Month X, XXXX (Doc. ID XXXXX); published Month X, XXXX

\begin{abstract}
Optical properties of natural photonic structures can inspire material developments in diversified areas, where spectral design of surfaces for camouflage is one. Here, reflectance, scattering and polarization properties of the cuticle of the scarab beetle Cyphochilus insulanus are studied with Spectral Directional Hemispherical Reflectance (SDHR), Bidirectional Reflection Distribution Function (BRDF) measurements and Mueller-Matrix Spectroscopic Ellipsometry (MMSE). At normal incidence a reflectance $(0.6-0.75)$ is found in the spectral range $400-1600 \mathrm{~nm}$ and a weaker reflectance $<0.2$ in the UV range as well as for wavelengths $>1600 \mathrm{~nm}$. A whiteness of $W=42$ is observed for mainly the elytra of the beetle. Chitin is a major constituent of the insect cuticle which is verified by the close similarity of the measured IR spectrum to that of $\alpha$-chitin. The BRDF signal shows close to Lambertian properties of the beetle for visible light at small angles of incidence. From the MMSE measurement it is found that the beetles appear as dielectric reflectors reflecting linearly polarized light at oblique incidence with low gloss and low degree of polarization. The measured beetle properties are properties that can be beneficial in a camouflage material.
\end{abstract}

OCIS codes: (120.5700) Reflection, (120.5820) Scattering measurements, (160.4760) Optical properties, (260.5430) Polarization, (260.2130) Ellipsometry and polarimetry; (330.1730) Colorimetry; http://dx.doi/org/10.1364/AO.99.099999

\section{Introduction}

Already in 1665 Hooke described observations of structural colors in nature in his book "Micrographia" [1]. Several scientists have since that time studied the optical properties of natural structures for various purposes. In some studies the objective is to describe and understand the role of these properties in nature [2-4], and in other studies the goal is to find inspiration for new applications [5-6] or to improve functionality in artificial devices by mimicking structures found in nature. Photonic crystals or other periodic dielectric structures are commonly found in nature and have potential to be used in optical devices. Structures in butterfly wings have, for example, been used as templates to large area periodic $\mathrm{ZrO}_{2}$ structures for potential applications and integration in optics technology [7]. Another example is the reflecting scales on wings of the butterfly Argyrophorus argenteus which are broadband reflectors. In this case, scaled-up replicas with thickness $<1 \mu \mathrm{m}$ have been manufactured and investigated using microwaves [8].

The main purpose of camouflage is to accomplish a look-alike effect as compared to the background, mainly in the visible and near infrared range, in order to minimize the signature as well as the detectability. Camouflage properties therefore have to be discussed in relation to application purpose, the sensors from which to hide and, the background property, as requirements varies with these. In the infrared range however, low emissive properties from highly diffuse reflection characteristics could be beneficial. Often diffuse reflection (scattering) and low angle dependence with a low degree of polarization are considered good properties for military 
camouflage, further described by Andersson et al. [9]. If generalizing, many natural objects are unpolarized, while manmade objects often have specific polarizing properties. Depolarizing properties could help to not reveal man-made surfaces. In the infrared (IR) $(3-12 \mu \mathrm{m})$ high reflectivity is often useful to conceal blackbody radiation from hot objects.

In this report we describe and discuss measurements of optical properties of natural structures. This is a first step towards the development of camouflage applications inspired by diffusively reflecting natural photonic structures. The function in nature of camouflage patterns has generated renewed interest [10]. For instance, the structures of cicada wings are suggested to have a camouflage-like anti-reflection function, [11] and inorganic replicas of these structures for use in solar cell applications are now emerging [12]. Other interesting candidates for camouflage studies are cuticle structures of beetles of the genus Cyphochilus, due to the combination of low weight, low gloss and high whiteness, which could be useful in both visual (VIS) and IR camouflage applications. These beetles have been investigated [13-15] and inspired production of thinner paper coatings with maintained whiteness [16] and production of fabric with increased whiteness [17]. Earlier studies were performed on single ( $5 \mu \mathrm{m}$ thick) scales from the cuticle. Key factors for the origin of the brilliant white appearance were found to be the width $(250 \mathrm{~nm})$ of a non-absorbing randomly disordered internal filamentary structure and the packing density corresponding to volume occupancy of $70 \%$ [13]. With this combination the optical spacing appear to be optimized for light scattering. The effects from optical crowding are thereby limited, i.e. the radiation fields of individual scattering zones dominate, instead of adopting the characteristics of larger ensembles. Optical crowding occurs when scattering zones come too close to each other, causing neighbour interaction [13]. To do the opposite, defining individual scattering centres in an amorphous network, is not possible [15]. Burresi et al. [15] have shown evidence of multiple scattering within the scales, which means that diffusion theory can be applied for scale modelling of optical properties.

Ellipsometry has recently been used to study scarab beetles [2-3, 18-20], and polarization properties have been reported. However, the beetles used in these studies are different from $C$. insulanus as they have a large specular reflection and low scattering. A comparison with these beetles is therefore not relevant. The previous studies of beetles from genus Cyphochilus are not species specific, and are mainly presenting reflectance data and do not include optical properties in terms of polarization and scattering. The latter information is valuable for development of camouflage

In this study the cuticle of Cyphochilus insulanus is explored with reflectance measurements which go further into the ultraviolet (UV) and IR ranges compared to previous studies. In addition, the important aspects of scattering and polarization properties of the cuticle are addressed using scatterometry and Mueller-matrix spectroscopic ellipsometry.

\section{Experimental Details}

Studies were performed on two specimens of Cyphochilus insulanus (Moser, 1918). Specimen A, (see Fig. 1.) a loan from the Swedish Museum of Natural History, has a length of $28 \mathrm{~mm}$. Specimen B, a loan from the Berlin Museum of Natural History, has a length of $20 \mathrm{~mm}$. Both specimens originate from Taiwan. Most of the measurements were performed on specimen $A$ but a selection of Mueller matrix ellipsometry measurements was also performed on specimen B for reference. The samples contained some small dark areas where scales were probably damaged or missing due to mechanical wear or age.

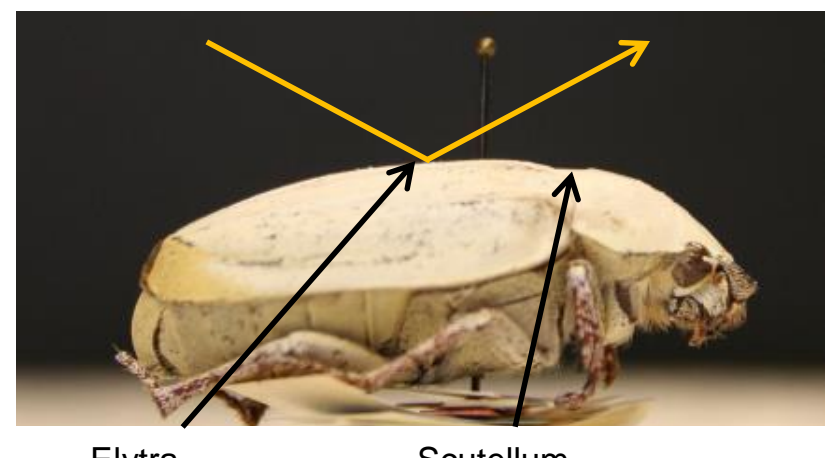

Elytra

Scutellum

Fig. 1. Photo of $C$. insulanus showing the direction of light during measurements, here reflected on the elytra.

The measurement methods used in this study were nondestructive and no sample preparation was needed. The authors acknowledge the complex reflecting structures of a target from nature, such as the beetle of this study. Despite of this a reflection could be divided into a specular and a diffuse component, which also is done here. Light diffusely reflected from a rough surface is scattered in several directions, whereas specularly reflected light from a smooth surface, like a mirror, is reflected with the same angle as the angle of incidence. A rough surface is considered matte whereas a smooth surface is considered glossy. However, a real surface can hardly ever be regarded as a perfectly diffuse scatterer or perfectly specular reflector; thus, there will to different degrees be a mixture of both components. A sum of the diffuse and the specular component is referred as a total reflection. In this study, three techniques are employed to optically characterize the beetle cuticle: Spectral Directional Hemispherical Reflectance (SDHR); scatterometry, measuring the reflectance as a function of angle, in the form of Bidirectional Reflection Distribution Function (BRDF) measurements; and Mueller-Matrix Spectroscopic Ellipsometry (MMSE). The experimental quantities obtained are the reflectance $R_{\mathrm{SDH}}$, the distribution function $f_{\mathrm{BRD}}$ and the Mueller matrix $\mathbf{M}$, respectively. The instrument setups were different for the three measurements in terms of orientation of the beetles. In the SDHR and BRDF measurements, the beetle was illuminated mainly on the elytra, i.e. the upper and flattest part of the cover wings in the direction from the back to the front of the beetle as shown in Fig. 1. 
These measurements were only performed on specimen A. The beam diameters in the BRDF measurements were approximately $1.5 \mathrm{~mm}$ and $4 \mathrm{~mm}$ at the wavelengths $633 \mathrm{~nm}$ and $3.39 \mu \mathrm{m}$, respectively. The MMSE measurements were performed using focusing optics to increase the irradiance of the specular reflection. This is the common measurement setup for beetle studies [21]. A spot size at normal incidence of less than $100 \mu \mathrm{m}$ was attained on both specimens on the elytra as well as on the scutellum (a triangular formed area between the wing-cases).

The SDHR measurement data, the reflectance $R_{\mathrm{SDH}}$, describes the relation between the irradiance of light incident from a specific direction and the total reflected radiation from a surface. The SDHR measurements were performed using two different systems with integrating spheres. In UV, VIS, and near-infrared (NIR) a Cary 5G system with a beam size of $5 \times 20 \mathrm{~mm}$ and a $150 \mathrm{~mm}$ diameter sphere (Labsphere DRA-CA-50D) coated with Spectralon ${ }^{\circledR}$ was used. In IR a Bruker IFS 55 FTIR system with a beam size of $5 \times 5 \mathrm{~mm}$ and a $75 \mathrm{~mm}$ diameter sphere (Bruker A 562) coated with diffusely reflecting gold was used. A schematic drawing of the experimental setup is seen in Fig.2a. Measurements were performed in the wavelength range $250-2500 \mathrm{~nm}$ at an angle of incidence of $10^{\circ}$ and in the wavelength range $2.44-25 \mu \mathrm{m}$ at an angle of incidence of $9^{\circ}$. The instruments were calibrated against a Spectralon $\AA$ diffuse reflectance standard with a reflectance of 99\% (UV-NIR) and a diffusely reflecting gold surface with a reflectance of $\sim 95 \%$ (IR).

The reflectance data $R_{\mathrm{SDH}}$ were used to calculate the CIE $L^{*} a^{*} b^{*}$ values of the $3 \mathrm{D}$-color space determined by the International Commission on Illumination [22]. $L *$ is a measurement of lightness used to subjectively describe human perception of brightness along a light-dark axis, $a$ *indicates the position at the red-green opponent axis, where positive numbers indicate red color, and $b^{*}$ gives the distinction between yellow and blue, where positive numbers indicate yellowness. The tristimulus values $X, Y, Z$ are relating the color coordinates to the perceived color. The whiteness $W$ can be calculated using $W=Y-1700\left(y-y_{n}\right)-800\left(x-x_{n}\right)$ according to a CIE standard for comparing color values [22] where $x, y$ are a ratio of the tristimulus values $X, Y, Z$ of the sample in relation to the sum of the three, and $x_{n}, y_{n}$ are the chromaticity coordinates of a perfect diffuser [22].

The BRDF describes the bidirectional reflectance at all incoming $\theta_{i}, \phi_{i}$ and outgoing $\theta_{r}, \phi_{r}$ angles, as a ratio between the scattered radiance $I_{r}$ and the incident irradiance $I_{i}$. The BRDF measurements were performed at the specified wavelengths, using a TASC Polarimetric scatterometer (TMA Technologies, Inc.) instrument. The measurements were performed over almost a semi-circle for scattering angles $-89^{\circ} \leq \theta_{r} \leq 89^{\circ}$ in the plane of incidence for incident angles $\theta_{i}=0^{\circ}, 20^{\circ}, 40^{\circ}, 60^{\circ}$ and $80^{\circ}$ as illustrated in Fig.2b. The azimuth angle was fixed during the measurements ( $\phi_{i}=180^{\circ}$ and $\phi_{r}=0^{\circ}$ in fig $2 \mathrm{~b}$ ). Both $\mathrm{s}^{-}$and p-polarized light where used, where $\mathrm{s}$ and $\mathrm{p}$ denote the electric field components perpendicular and parallel to the plane of incidence, respectively. a)

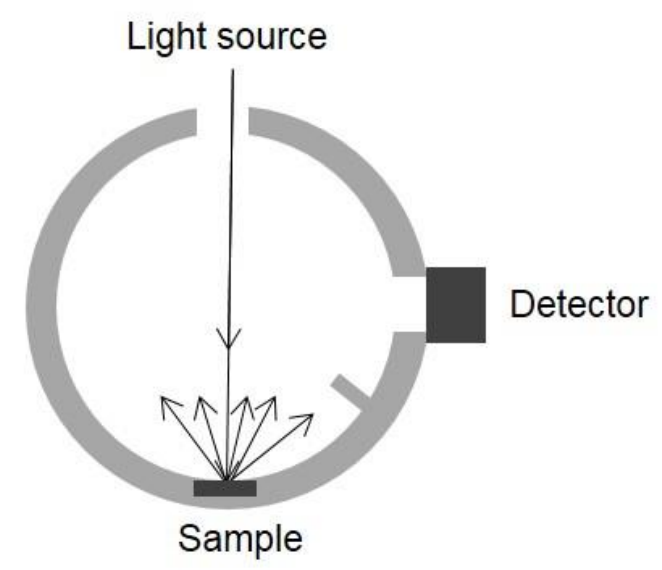

b) Reflected

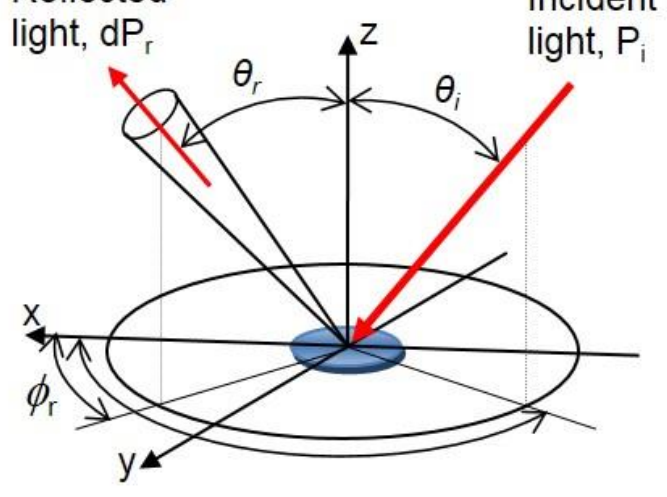

c)

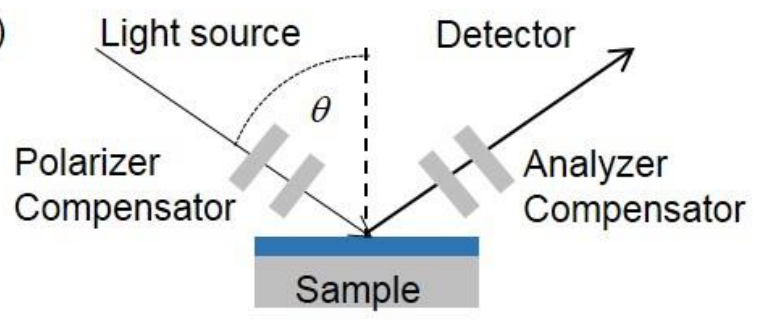

Fig. 2. Configurations of the measurement methods employed. a) Spectral Directional Hemispherical Reflectance (SDHR). b) Scatterometry (BRDF measurements) c) Mueller-matrix spectroscopic ellipsometry (MMSE).

The measured $f_{\mathrm{BRD}}$ has units of inverse steradians $\left[\mathrm{sr}^{-1}\right]$ and can be written as

$$
f_{\mathrm{BRD}} \approx \frac{\mathrm{d} P_{r} / \mathrm{d} \Omega_{r}}{P_{i} \cos \theta_{r}} \approx \frac{P_{r} / \Omega_{r}}{P_{i} \cos \theta_{r}}
$$

where $P_{i}$ is the incident radiant flux on the surface, $\mathrm{d} P_{r}$ is the light flux scattered into a solid angle $\mathrm{d} \Omega_{r}$ [23-25]. The factor $\cos \theta_{r}$ adjust the illuminated area $A$ to the corresponding size viewed from the scatter direction. (In Eq. (1) the polarization and wavelength 
dependence are suppressed and the dependence of the illuminated area $A$ on the sample surface is eliminated for simplification). The value of $f_{\mathrm{BRD}}$ can be described as the directional reflectance per unit solid angle of the collected scattered light [25]. Prior to the measurements, a calibration was performed according to a standard procedure [25] by directly measuring the power from the radiation source (laser) upon the sample. In addition, the accuracy of the measurements was controlled using near Lambertian Spectralon reference surfaces with different reflectance levels. A Lambertian surface follows Lambert's law $I\left(\theta_{r}\right)=I_{0} \cos \theta_{r}$ stating that the irradiance of light scattered from a specific point follow a cosine characteristic, where $\theta_{r}$ is the polar angle of the scattered light and $I_{0}$ is the incident irradiance at the point. That is, a Lambertian surface is ideally diffuse where the irradiance of the reflected light is the same regardless of the viewing angle. In other words $f_{\mathrm{BRD}}$ is constant for all angles $\theta_{r}$ [25]. If $R_{\mathrm{SDH}}$ is known for a specific wavelength $\lambda, f_{\mathrm{BRD}}$ for an ideal Lambertian surface can be calculated from the relation $f_{\mathrm{BRD}}=R_{\mathrm{SDH}}(\lambda) / \pi$. Worth mentioning is that, no ideal Lambertian surfaces exist, and also surfaces with close to Lambertian properties are rare in nature.

In the MMSE measurements the polarization change of the specularly reflected beam is measured. The small samples which are curved and inhomogeneous require a focused beam, whereby a small beam size is obtained. Measurements were performed on the scutellum (instead of the elytra) and an area completely covered with scales was selected. The scales dimensions are much smaller than the size of the beam spot. Measurements were performed in the wavelength range $250-1000 \mathrm{~nm}$ in steps of $1 \mathrm{~nm}$ at angles of incidence between $20^{\circ}$ and $75^{\circ}$ in steps of $5^{\circ}$ using a dual rotating compensator ellipsometer (RC2, J.A. Woollam Co., Inc.) as illustrated in Fig.2c.

In the Stokes-Mueller formalism, the Stokes vectors $\mathbf{S}_{i}$ and $\mathbf{S}_{o}$ describe the incoming and outgoing light, respectively, and the $4 \times 4$ matrix M, with 16 elements $M_{a b}(a, b=1,2,3,4)$, describes the sample. These parameters are related through the relation

$$
\begin{aligned}
\mathbf{S}_{o}= & \left(\begin{array}{l}
I_{0} \\
Q_{0} \\
U_{0} \\
V_{0}
\end{array}\right)= \\
& =\left(\begin{array}{llll}
1 & m_{12} & m_{13} & m_{14} \\
m_{21} & m_{22} & m_{23} & m_{24} \\
m_{31} & m_{32} & m_{33} & m_{34} \\
m_{41} & m_{42} & m_{43} & m_{44}
\end{array}\right)\left(\begin{array}{c}
I_{i} \\
Q_{i} \\
U_{i} \\
V_{i}
\end{array}\right)=\mathbf{M S}_{i}
\end{aligned}
$$

where the Mueller matrix $\mathbf{M}$ is normalized according to $m_{a b}=$ $M_{a b} / M_{11}$. The $Q$ and $U$ elements are irradiances for the part of the light being linearly polarized with preference for $x$ or $y$ and $+45^{\circ}$ or $-45^{\circ}$ respectively, $V$ is the left- or right-handed circular polarization preference and $I$ is the total irradiance. Often $\boldsymbol{S}_{i}$ is normalized with $I_{i}$ whereby $I_{i}=1$.

In the present study $\mathrm{s}^{-}, \mathrm{p}^{-}$and unpolarized incoming light are considered. The incident normalized Stokes vector for $\mathrm{p}^{-}$and $\mathrm{s}^{-}$ polarized light are here defined as $\mathbf{S}_{p i}=\left(\begin{array}{llll}1 & 1 & 0 & 0\end{array}\right)^{T}$ and $\mathbf{S}_{s i}=$ $\left(\begin{array}{llll}1 & -1 & 0 & 0\end{array}\right)^{T}$, respectively, and for incident unpolarized light as $\mathbf{S}_{u i}=\left(\begin{array}{llll}1 & 0 & 0 & 0\end{array}\right)^{T} \quad(T$ indicates transpose $)$. The degree of polarization $P$ is defined as $P=\left(Q^{2}+U^{2}+V^{2}\right)^{1 / 2} / I$. When using normalized Muller-matrix elements $m_{a b}$ the degree of polarization of reflected light for incoming unpolarized, $\mathrm{s}^{-}$and p-polarized light are denoted $P_{u}, P_{s}$, and $P_{p}$ respectively, and are defined as

$$
\begin{aligned}
& P_{u}=\left[\mathrm{m}_{21}{ }^{2}+\mathrm{m}_{31}{ }^{2}+\mathrm{m}_{41}{ }^{2}\right]^{1 / 2} \\
& P_{s}=\frac{\left[\left(\mathrm{m}_{21}-\mathrm{m}_{22}\right)^{2}+\left(\mathrm{m}_{31}-\mathrm{m}_{32}\right)^{2}+\left(\mathrm{m}_{41}-\mathrm{m}_{42}\right)^{2}\right]^{1 / 2}}{1-m_{12}} \\
& P_{p}=\frac{\left[\left(m_{21}+m_{22}\right)^{2}+\left(m_{31}+m_{32}\right)^{2}+\left(m_{41}+m_{42}\right)^{2}\right]^{1 / 2}}{1+m_{12}}
\end{aligned}
$$

A polarization state can be defined by the ellipticity $e$ and the azimuth $\alpha$ of the polarization ellipse [26]. The ellipticity is given by $e=b / a$, where $a$ and $b$ are the major and minor axis, respectively, of the polarization ellipse. The ellipticity has values in the range $-1 \leq e \leq 1$, where 0 corresponds to linearly polarized light and $+1 /-1$ to circularly polarized light with right-/left-handed polarization. The azimuth $a$ is the angle between the major axis of the ellipse and the $\mathrm{p}$-polarization direction and defined in the range $-90^{\circ} \leq \alpha<90^{\circ}$. It is used to describe the polarization direction. Using Stokes elements, these parameters can be written as

$$
\begin{aligned}
& e=\tan \left(\frac{1}{2} \arcsin \left[V /\left(Q^{2}+U^{2}+V^{2}\right)^{1 / 2}\right]\right) \\
& \alpha=\frac{1}{2} \arctan (U / Q)
\end{aligned}
$$

The software CompleteEASE (J.A. Woollam Co., Inc.) was used for data analysis and preparation of the MMSE results.

\section{Results and Discussion}

Figures 3 and 4 show $R_{\mathrm{SDH}}$ measured on specimen $\mathrm{A}$ as a function of $\lambda$ and $\mathrm{cm}^{-1}$ respectively for incident unpolarized light in the UVNIR $(250-2500 \mathrm{~nm})$ and in the IR $(2.44 \mu \mathrm{m}-25 \mu \mathrm{m})$ regions. The latter is presented versus wavenumber $\left(\mathrm{cm}^{-1}\right)$ in the range $4100-400 \mathrm{~cm}^{-1}$ which is common for practical reasons when reporting vibration bands, as is the case here, see also Table 1. 


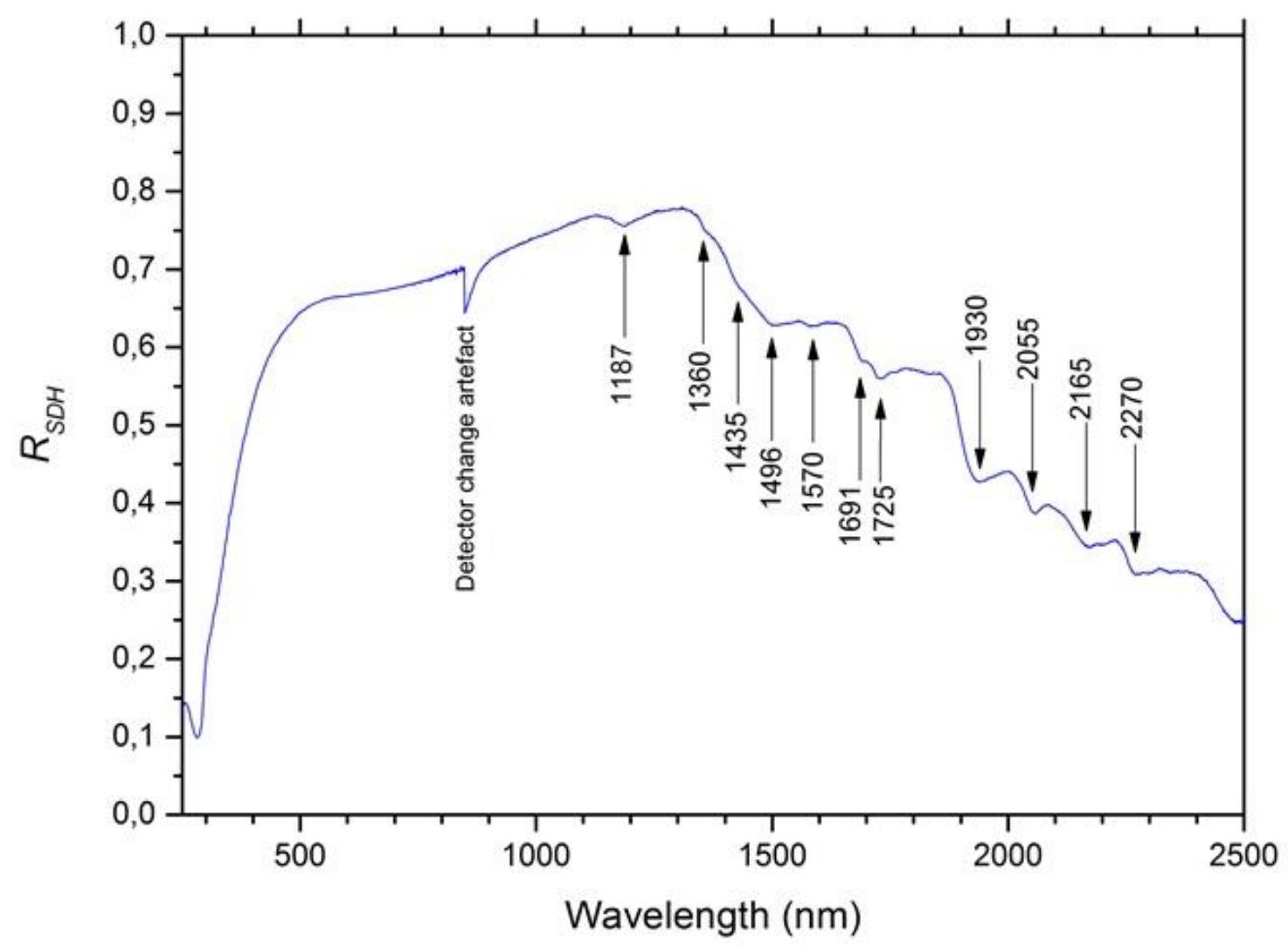

Fig. 3. Spectral reflectance $R_{\mathrm{SDH}}$ in the UV-VIS-NIR spectral range showing absorption bands of $1^{\text {st }}$ and $2^{\text {nd }}$ overtones and combination of bands as listed in Table 1.

In Fig. 3 an artefact caused by a difference in reference and sample surface appears due to a detector change at $850 \mathrm{~nm}$. This failure in calibration in a small range is not affecting any analyse or results. The general spectral appearance shows an overall strong reflectance, $0.5<R_{S D H}<0.8$ in the range $400-1850 \mathrm{~nm}$ with a maximum near $1300 \mathrm{~nm}$. Below $400 \mathrm{~nm}$ the reflectance is decreasing rapidly to a minimum of approximately 0.1 at $\sim 265 \mathrm{~nm}$. In the range 1300- $2500 \mathrm{~nm}$, a number of absorption bands can be observed. The reflectance in the VIS spectral region $(400-700 \mathrm{~nm})$ is around 0.5-0.7, with an almost constant reflectance level of 0.65 between 500 and $700 \mathrm{~nm}$, which is in accordance with the white appearance of the investigated beetles. Similar observations were made for a Cyphochilus specimen in the studies by Vukusic et al. [13], Burresi et al. [15] and Luke et al. [14]. Burresi et al. attained slightly higher reflectance in the visible $(0.75-0.8)$.

Both Burresi et al. and Luke et al. made additionally whiteness studies of other beetles (Lepidiota stigma and Calothyrza margaritifera) with similar results. Also Lafait et al. obtained comparable results in studies on the Calothyrza margaritifera beetle [27].

The obtained $L^{*} a^{*} b^{*}$ parameters and whiteness $W$ determined from $R_{\mathrm{SDH}}$ on specimen A are as follows: $L^{*}=84.5, a^{*}=-0.8$, $b^{*}=4.7, W=41.7$. These values indicate a pale-colored surface, with a tint to green-yellow color. The previously reported whiteness value of 60 , based on the optical response from a single scale of a Cyphochilus specimen [13], differs from the whiteness level $W=42$ found in this study. Our whiteness calculation is based on measurements with a large beam where nearly the whole length of one side of the elytra is illuminated. Thereby also areas where the scales are damaged or missing are included which will lower the whiteness level. With a value of $W>40$ the measured surface is still regarded as white according to the CIE whiteness equation [28].

Figure 4 shows that the reflectance in the IR-range is rather low and below 0.1 for wave numbers in the range $400-3700 \mathrm{~cm}^{-1}(25-$ $2.7 \mu \mathrm{m})$. A broad local maximum of 0.1 is observed near $2300 \mathrm{~cm}^{-1}$ $(4.3 \mu \mathrm{m})$. The maximum reflectance in the IR range is larger than 0.2 measured for wavenumbers larger than $3800 \mathrm{~cm}^{-1}(\lambda<2.6 \mu \mathrm{m})$. 
The positions of identified vibrational modes giving rise to the absorption bands are indicated in Fig.3 and Fig.4 and listed in Table 1 where associated functional groups and assignments are suggested. Fundamental vibration bands of $\mathrm{O}-\mathrm{H}, \mathrm{N}-\mathrm{H}, \mathrm{C}-\mathrm{H}, \mathrm{C}=\mathrm{O}$ and $\mathrm{C}-\mathrm{N}$ functional groups are dominating in the mid IR range, whereas overtones and combinational bands of these are positioned in the NIR. All of the amide bands are observed up to amide band VI [29]. The identities of the bands at $695 \mathrm{~cm}^{-1}, 645 \mathrm{~cm}^{-1}$ and $590 \mathrm{~cm}^{-1}$ are uncertain. The IR spectrum closely resembles that of $\alpha$-chitin [30] which is expected since chitin has been identified as a major constituent of the cuticle of scarab beetles and insects in general.

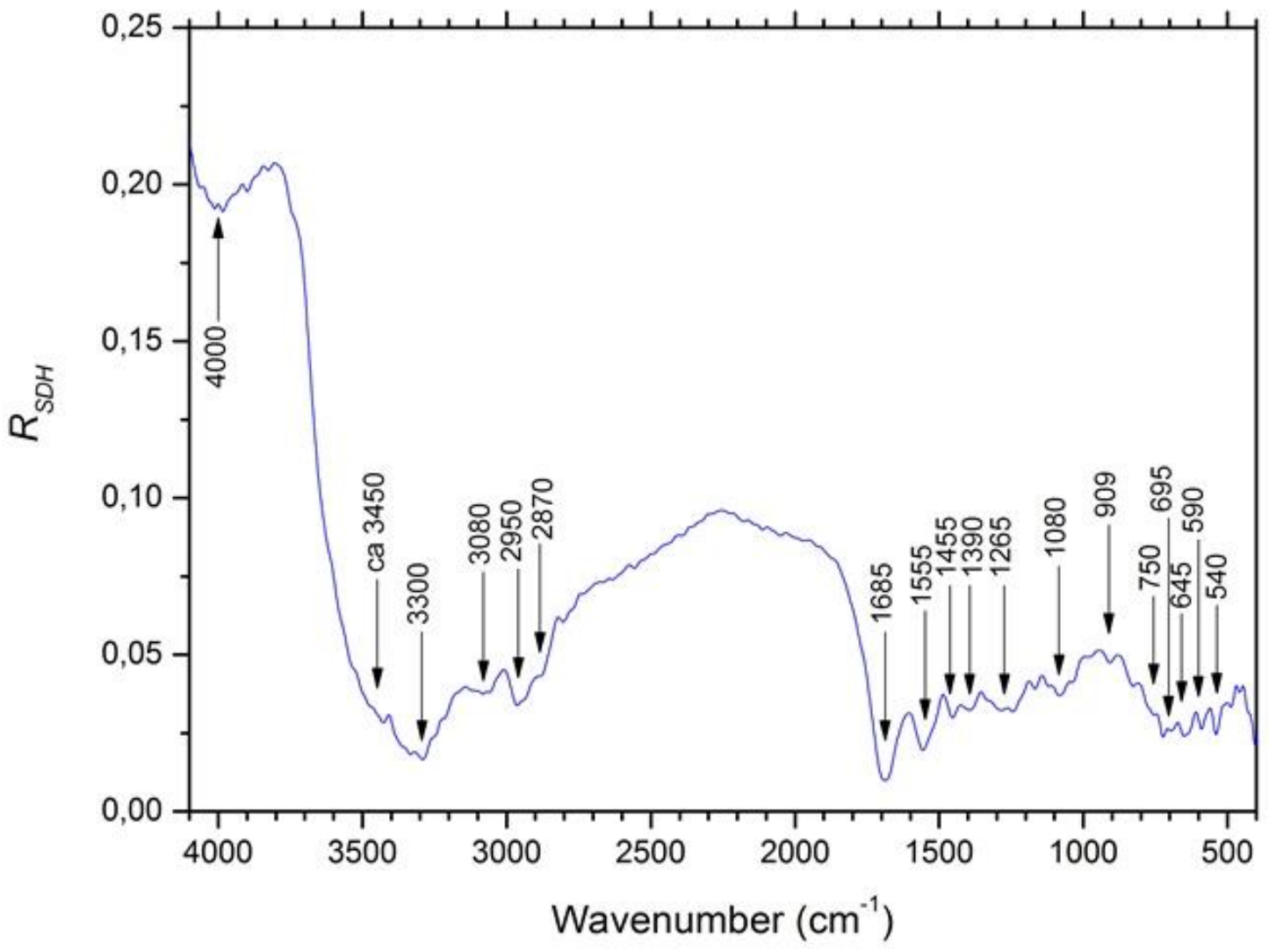

Fig. 4. Spectral reflectance $R_{\mathrm{SDH}}$ measurement in the IR region showing absorption bands corresponding to fundamental vibrational modes as listed in Table 1.

Figures 5 and 6 show results from the BRDF measurements on $C$. insulanus at multiple $\theta_{i}$ for $\mathrm{s}^{-}$and $\mathrm{p}$-polarized light. The scattering properties are here presented by $f_{\mathrm{BRD}}$ as a function of $\theta_{r}$ at $\lambda=633 \mathrm{~nm}$ and $\lambda=3.39 \mu \mathrm{m}$. The sharp minima seen when $\theta_{i}=$ $\theta_{r}$ are due to source shadowing in the measurement setup. For $\lambda=633 \mathrm{~nm}$ it can be noticed that for $\theta_{i}<40^{\circ}, f_{\mathrm{BRD}}$ is practically constant for both $\mathrm{s}^{-}$and $\mathrm{p}$-polarized light, that is, the beetle is nearly Lambertian [25] in this range. For $\theta_{i}=40^{\circ}$ and $\theta_{i}=60^{\circ}$ a small but broad peak appears due to specular reflection for angles around $\theta_{r}=50^{\circ}$ and $70^{\circ}$, respectively. This is more pronounced for $\mathrm{s}^{-}$ polarized light than for $\mathrm{p}$-polarized light and can be explained by the suppression of the reflection of the p-polarized light near the
Brewster angle. For $\theta_{i}=80^{\circ}, f_{\mathrm{BRD}}$ is increasing to values $>10 \mathrm{sr}^{-1}$ for $\theta_{r}>80^{\circ}$. In the studies of the beetle Calothyrza margaritifera having a white hairy structure, Lafait et al. performed wavelengthresolved scatterometry measurements and spatial mapping of scattered light [27].

Normalized $f_{\mathrm{BRD}}$ values of $0.5-0.65$ for $633 \mathrm{~nm}$ at $10^{\circ} \leq \theta_{r} \leq$ $60^{\circ}$ for $\theta_{i}=30^{\circ}$ were reported. In this study, measurements on $C$. insulanus give $0.1<f_{\mathrm{BRD}}<0.3 \mathrm{sr}^{-1}$ at corresponding wavelengths and angles. A direct comparison between these studies is difficult due to different measurement setups. The general conclusion is however the same; the white structures show near Lambertian properties, at least for small $\theta_{i}$ in the visible. This is an 
interesting finding as also close to Lambertian surfaces are scarce in nature [31].

For measurements at $3.39 \mu \mathrm{m}$, lower $f_{\mathrm{BRD}}$ values and pronounced peaks at near specular directions can be observed in general. At larger $\theta_{i} f_{\mathrm{BRD}}$ increases rapidly to levels $>1$ for large scattering angles. For $\theta_{i} \leq 40^{\circ}$ the values are low, particularly in the back scattering directions (negative angles). Again, slightly higher values of $f_{\mathrm{BRD}}$ are observed for s-polarized light compared to $\mathrm{p}$-polarized light, especially close to the Brewster angle. At $3.39 \mu \mathrm{m}$ the characteristic is clearly not Lambertian. However, many diffusely

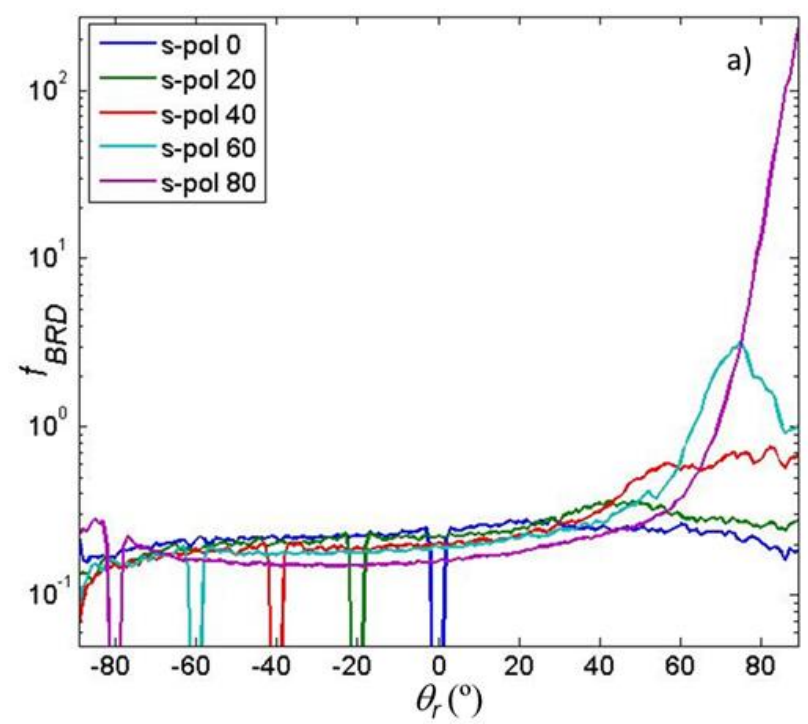

scattering samples are not Lambertian enough for the assumption $f_{\mathrm{BRD}}=R_{\mathrm{SDH}}(\lambda) / \pi$ to be valid. The $\sim 10^{\circ}$ displacement of the specular reflection peaks in relation to the incidence angle is due to the curved surfaces of the beetles and complicated alignment. The actual peak is rather wide, since the organization of the scales gives rise to a distribution of off-specular angle reflection, i.e. the surface normal is not well defined nor is the angle of incidence. However, these mismatches are not too crucial in this case as a specular reflection from a diffuse sample cannot be well defined.

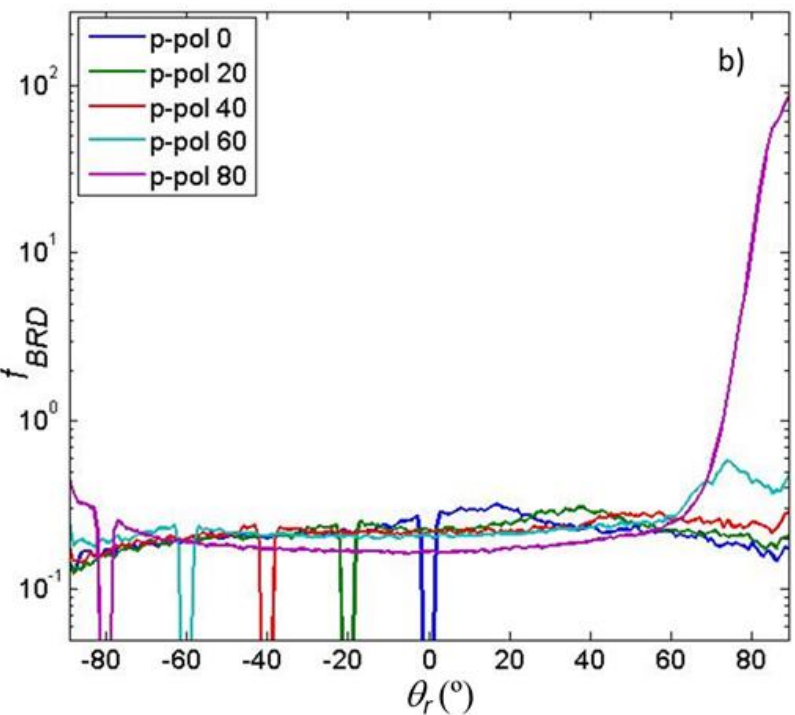

Fig. 5. The scattering properties of $C$. insulanus in terms of $f_{\mathrm{BRD}}$ as a function scattering angle $\theta_{r}$, for different incident angles $\theta_{i}$ and for a) s-polarized light and b) p-polarized light at wavelength $\lambda=633 \mathrm{~nm}$.
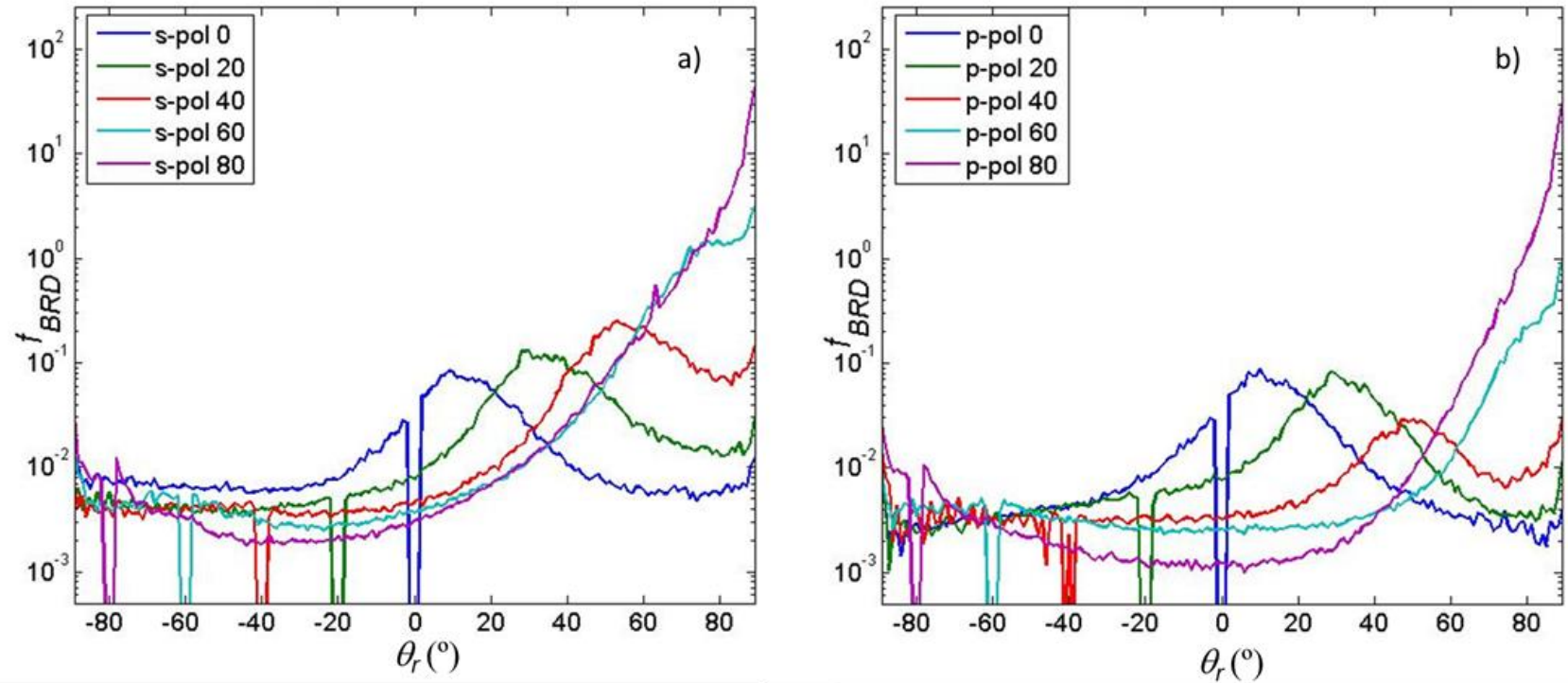

Fig. 6. The scattering properties of $C$. insulanus in terms of $f_{\mathrm{BRD}}$ as a function scattering angle $\theta_{r}$, for different incident angles $\theta_{i}$ and for a) s-polarized light and b) p-polarized light at wavelength $\lambda=3.39 \mu \mathrm{m}$. 
Both specular and diffuse scattering occur on this specimen. The thicknesses of the scales are $\sim 5 \mu \mathrm{m}$ [14] which are roughly of the same order as the wavelength in the IR measurement $(2.44-25 \mu \mathrm{m})$. On the other hand, the internal structure giving rise to the white feature has dimensions of the order of visible light $(400-700 \mathrm{~nm})$. However, the degree of specular scattering generally increases if the structure is small, and we do see a larger specular response in the case of IR radiation.

Figures 7 and 8 show results from MMSE experiments on the scutellum of specimen A. Measurements on the elytra (as reported for the SDHR and BRDF measurement) gave similar results. All measurements gave an ellipticity $e$ close to zero (not shown here), e.g. the polarized part of the reflected light is almost linearly polarized for all incidence angles, wavelengths and polarization states.

Figure 7 shows the degree of polarization at $\theta_{i}=20^{\circ}$ and $75^{\circ}$ for the incoming $\mathrm{u}^{-}, \mathrm{s}^{-}$and $\mathrm{p}$-polarizations. In these plots the variations with wavelength are seen to be small. In Fig. 8. polar $\lambda \theta-$ contour plots show the polarization direction in terms of the azimuth angle $\alpha$ as well as the degree of polarization $P_{u, s, p}$ for incoming unpolarized, s-polarized and p-polarized light as determined from Eqs. 3-5.

In Fig. 8a it is seen that for incident unpolarized light the azimuth angle $\alpha$ is rather stable at values near $90^{\circ}$ for most incident angles $\theta_{i}$, corresponding to s-polarized reflection. Fluctuations towards smaller $\alpha$ are seen for smaller $\theta_{i}\left(20^{\circ}-30^{\circ}\right)$ for most wavelengths. The degree of polarization diminishes with decreasing $\theta_{i}$ and values $P_{u}<0.1$ are attained for $\theta_{i}<30^{\circ}$. The largest values of $P_{u} \sim 0.45$ are attained for large $\theta_{i}$.

In Fig. $8 \mathrm{~b}$ it is seen that for incident s-polarized light, $\alpha$ is $\sim 90^{\circ}$ for all $\theta_{i}$ and $\lambda$, corresponding to s-polarized reflection. Large $P_{S}$ around 0.9 is attained for large $\theta_{i} . P_{S}$ decreases gradually with decreasing $\theta_{i}$ to values $\sim 0.2$.

In Fig. $8 \mathrm{c}$ it is seen that for incident p-polarized light, $\alpha$ is close to zero for all $\theta_{i}$ and $\lambda$, which indicates p-polarized reflection. Fluctuations of azimuth up to $\sim 20^{\circ}$ for $\theta_{i}$ in the range $30^{\circ}-60^{\circ}$ are found for several wavelengths. The highest values of $P_{p} \sim 0.75$ are attained for large $\theta_{i}$ at all $\lambda$. The value diminishes rapidly to $P_{p} \sim 0.15$ with decreasing $\theta_{i}$.

In Fig. 8, the degree of polarization is presented together with the azimuth of polarization $(\alpha)$. The latter, however, only concerns the fraction of light that is polarized. When $P$ becomes small, like for small incident angles in Fig. 8a, the determination of azimuth becomes uncertain and fluctuations are seen.

The Brewster angle $\theta_{B} \sim 58^{\circ}$ at $633 \mathrm{~nm}$ was determined from $n$ of chitin [32] and could possibly influence the degree of polarization as mainly s-polarized light will be reflected at that angle.

The MMSE results show that $P$ in general increases with $\theta_{i}$ but the state of polarization does not change considerably upon reflection. However, for small $\theta_{i}$ the beetle is depolarizing and only a small fraction of the light is polarized after reflection. The azimuth angle $\left(90^{\circ}\right)$ indicates linearly s-polarized response for unpolarized and s-polarized incoming light, but linearly p-polarized response for
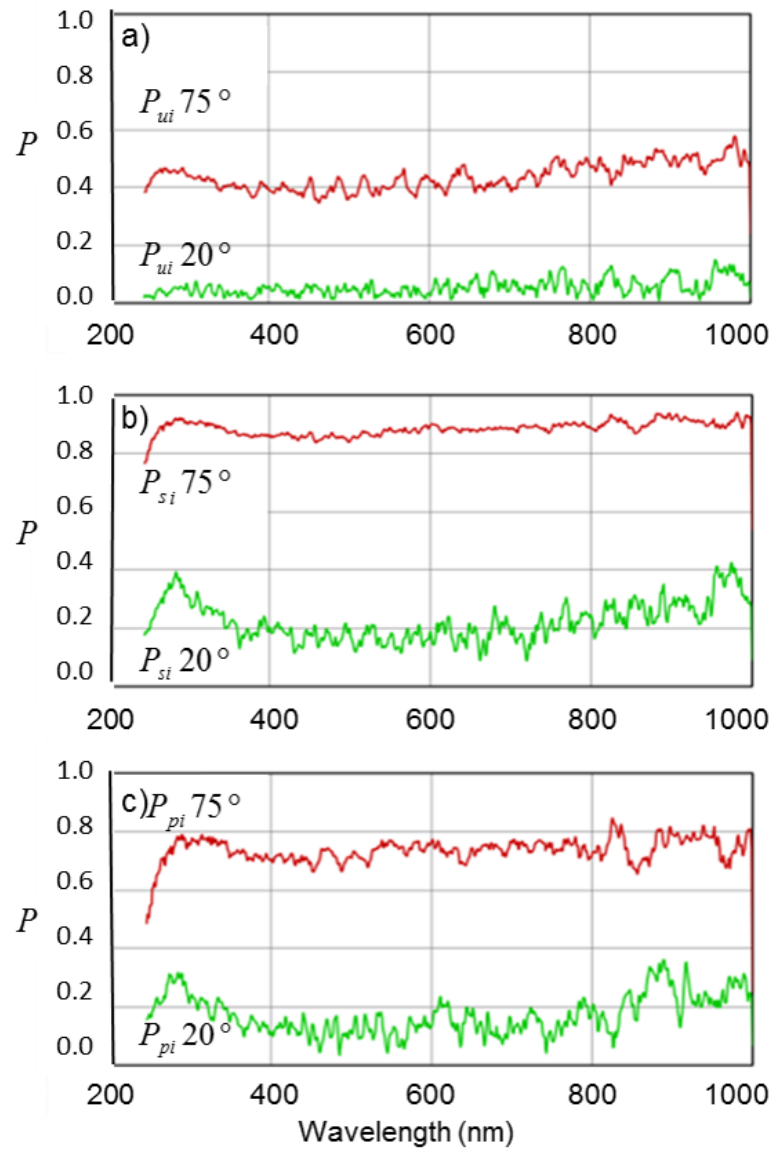

Fig. 7. Degree of polarization $P$ versus wavelength for incident angles $\theta=20^{\circ}$ and $75^{\circ}$ for a) incoming unpolarized light, b) $\mathrm{s}^{-}$ polarized and c) p-polarized light.

incoming p-polarized light $\left(0^{\circ}\right)$.

The result in Figs. 7 and 8 exhibit noise-like features. However, it is not clear if these features are noise-related. The instrument used has a noise level in $m_{a b}$ which is more than one order of magnitude lower than the variations observed. In principle noise may propagate in the data analysis and can be amplified in some derived parameters. However, if we take $P_{u}$ in Fig. $7 \mathrm{a}$ as an example, we find from Eq. 3 that $P_{u}$ is almost equal to $m_{21}$ as $m_{31}$ and $m_{41}$ are very small. The expected instrument noise in $m_{21}$ is much smaller than the variations seen in Fig. 7a and these variations may thus very well originate from spectral variations in reflectance from the sample. Interference oscillations across the cuticle as observed and analyzed for Cotinis mutabilis [33] could be one possible explanation. The weak signatures seen in the $C$. insulanus data compared to in C. mutabilis, may be due to that the cuticle is composed of scales increasing the inhomogeneity and thus heavily distorting interference oscillations. Without further studies it is, however, not possible to fully explain the origin of the observed spectral variations in Fig. 7. The azimuth variations in Fig. 8 are more likely noiserelated, especially for unpolarized incident light. We see from Eq. 7 
that alpha is dominated by $m_{31}$ in this case and $m_{31}$ is very small and thus has a low signal to noise ratio. In other words, the uncertainty in the determination of the direction of linear polarization becomes large when the degree of polarization is small.

Hodgkinson et al. [19] have done polarization studies on the narrow-band reflecting green and red beetle Stephanorrhina guttata a)
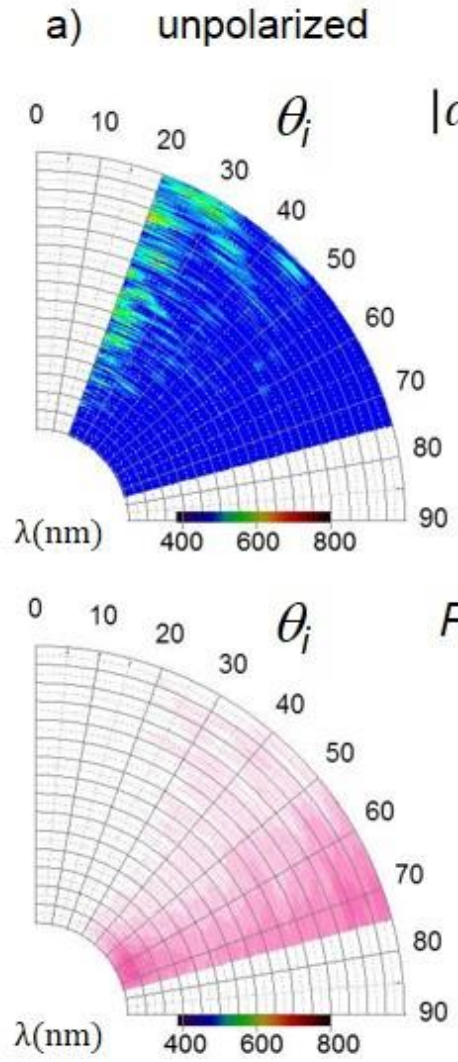

b) s-polarized
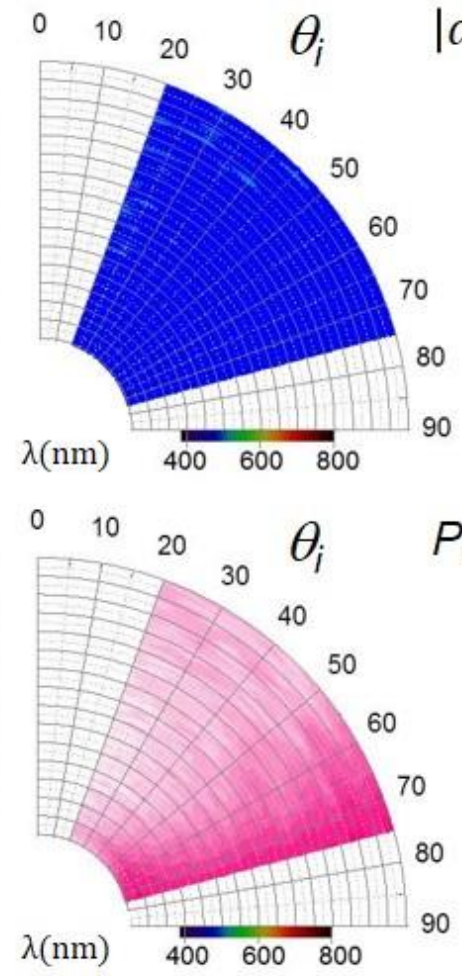

c)
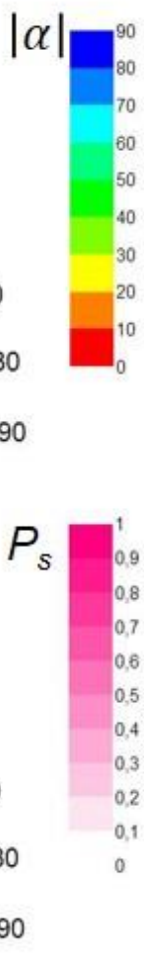
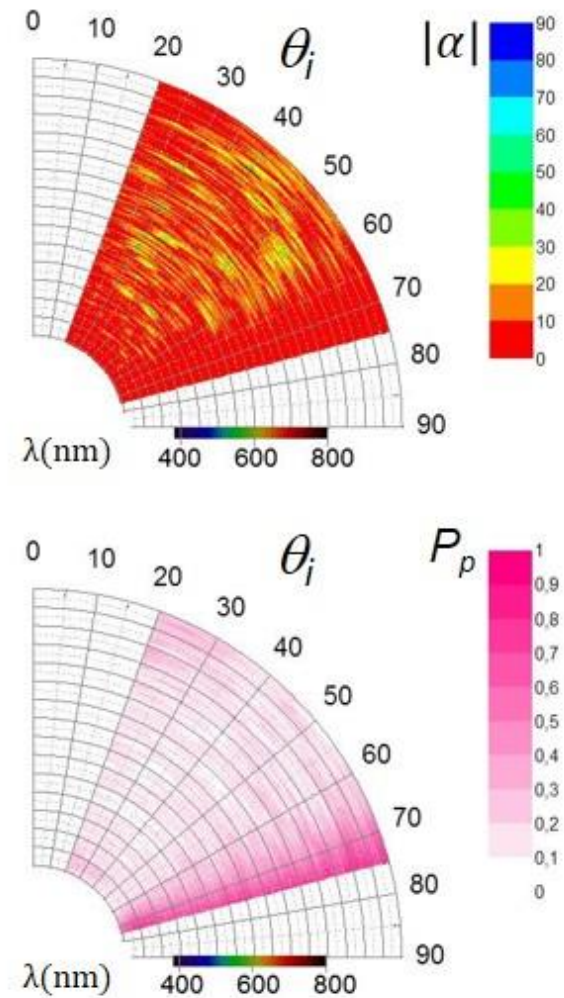

Fig. 8. Azimuth $\alpha$ and degree of polarization $P$ derived from Mueller-matrix data measured on $C$. insulanus. Results are shown for a) incoming unpolarized, b) s-polarized c) and p-polarized light as a function of wavelength $\lambda$ and incident angle $\theta_{i}$.

\section{Conclusions}

Cyphochilus insulanus, has a reflectance that is high $(\sim 0.6)$ but diffuse in the VIS and NIR spectral ranges and low in the long wave IR range. The IR measurements show absorption bands characteristic of $\alpha$-chitin. Near Lambertian scattering was found in the visible range for small $\theta_{i}<40^{\circ}$ and diffuse scattering properties with a specular scent in the visible for larger $\theta_{i}$ and in IR for all incident angles. The whiteness level attained was 42 , still giving a white appearance, as measured on the beetle as whole, but different from results on single scales found in the literature. In general, the polarized part of the reflected light from $C$. insulanus is linear for all wavelengths and incident angles. The azimuth angle is almost independent of wavelength implying s-polarized response for unpolarized and s-polarized incident light and with a p-polarized response for incoming $\mathrm{p}$-polarized light. The degree of polarization increases with the angle of incidence.
We conclude that the beetle surface consisting of scales on a cuticle reflects like a dielectric material, has low gloss as a consequence of the diffuse scattering properties, low dispersion, no specific polarization signature, all suitable properties for camouflage applications. The structure appears to be optimized for scattering in the visible spectral range. There is no reason to believe that the diffuse scattering properties, received for $633 \mathrm{~nm}$, should differ greatly in the rest of the visible range, since the spectrum of Fig. 4. is rather flat. The sample show linear polarizing properties as it preserves the polarization of the incident light, except for small incident angles at which only a small amount of the light is polarized, i.e. the beetle is depolarizing.

\section{Acknowledgements}

This work has been financially supported by the Swedish Armed Forces through the Research and Technology program (ref.nr. 
AF.922.0409) and the Swedish Government Strategic Research Area in Materials Science on Functional Materials at Linköping University (Faculty Grant SFO-Mat-LiU \# 2009-00971). The Knut and Alice Wallenberg foundation is acknowledged for support to instruments. The Swedish Museum of Natural History and the Berlin Museum of Natural History are acknowledged for loan of beetles. Dr. Anna Pohl at FOI is acknowledged for calculating the CIE coordinates.

\begin{tabular}{|c|c|c|c|c|}
\hline \multicolumn{2}{|c|}{ Spectral position } & \multirow[t]{2}{*}{ Group } & \multirow[t]{2}{*}{ Description } & \multirow[t]{2}{*}{ Assignment } \\
\hline $\mathrm{nm}$ & $\mathrm{cm}^{-1}$ & & & \\
\hline 1187 & 8424 & $\mathrm{CH}_{3}$ & C-H stretching $2^{\text {nd }}$ overtone [34] & \\
\hline 1360 & 7353 & $\mathrm{CH}_{3}$ & C-H combination [34] & \\
\hline 1435 & 6969 & $\mathrm{CH}_{2}$ & C-H combination [34] & \\
\hline 1496 & 6684 & $\mathrm{~N}-\mathrm{H}$ & $\mathrm{N}-\mathrm{H}$ stretching $1^{\text {st }}$ overtone [34] & Amide A \\
\hline 1570 & 6369 & $\mathrm{~N}-\mathrm{H}$ & $\mathrm{N}-\mathrm{H}$ stretching $1^{\text {st }}$ overtone [34] & \\
\hline 1691 & 5914 & $\mathrm{CH}_{3}$ & C-H stretching $1^{\text {st }}$ overtone [34] & \\
\hline 1725 & 5797 & $\mathrm{CH}_{2}$ & $\mathrm{C}-\mathrm{H}$ stretching $1^{\text {st }}$ overtone [34] & \\
\hline 1930 & 5181 & $\mathrm{O}-\mathrm{H}$ & $\mathrm{O}-\mathrm{H}$ stretching/HOH deformation combination [34] & $\begin{array}{l}\text { Starch/ } \\
\text { cellulose }\end{array}$ \\
\hline 2055 & 4866 & & $\begin{array}{l}\text { Symmetric N-H stretching/Amide I ( } \mathrm{C}=\mathrm{O} \text { stretching) } \\
\text { combination, and/or N-H/Amide II or III combination [34] }\end{array}$ & Protein \\
\hline 2165 & 4619 & $\mathrm{HC}=\mathrm{CH}$ & $\begin{array}{l}\text { Asymmetric C-H stretching/C-H deformation combination } \\
\text { of } \mathrm{HC}=\mathrm{CH}[34]\end{array}$ & \\
\hline 2270 & 4405 & $\mathrm{O}-\mathrm{H} / \mathrm{C}-\mathrm{O}$ & O-H stretching/C-O stretching combination [34] & Cellulose \\
\hline 2500 & 4000 & $\mathrm{C}$ & $\mathrm{C}-\mathrm{H}$ stretching/C-C and C-O-C stretching [34] & Starch \\
\hline 2898 & 3450 & $\mathrm{O}-\mathrm{H}$ & O-H stretching $[30,35]$ & \\
\hline 3030 & 3300 & $\mathrm{~N}-\mathrm{H}$ & $\mathrm{N}-\mathrm{H}$ stretching $[29-30]$ & Amide A \\
\hline 3250 & 3080 & $\mathrm{~N}-\mathrm{H}$ & $\mathrm{N}-\mathrm{H}$ stretching $[29]$ & Amide B \\
\hline 3380 & 2950 & $\mathrm{C}-\mathrm{H}$ & C-H stretching $[30,35]$ & \\
\hline 3480 & 2870 & $\mathrm{C}-\mathrm{H}$ & C-H stretching $[30,35]$ & \\
\hline 5900 & 1686 & $\mathrm{C}=\mathrm{O}$ & $\mathrm{C}=\mathrm{O}$ stretching $[29-30]$ & Amide I \\
\hline 6400 & 1555 & & $\mathrm{C}-\mathrm{N}$ stretching $+\mathrm{N}-\mathrm{H}$ bending [29-30] & Amide II \\
\hline 6900 & 1455 & & $\begin{array}{l}\mathrm{CH}_{2}+\mathrm{CH}_{3} \text { bending }[35] \\
\mathrm{CH}_{2} \text { bending and } \mathrm{CH}_{3} \text { deformation [30] }\end{array}$ & \\
\hline 7200 & 1390 & $\mathrm{CH}_{3}$ & $\mathrm{CH}_{3}$ bending [35] & \\
\hline 7900 & 1265 & $\mathrm{~N}^{-}$ & $\mathrm{C}-\mathrm{N}$ stretching $+\mathrm{N}-\mathrm{H}$ bending $[29]$ & Amide III \\
\hline 9259 & 1080 & $\mathrm{C}-\mathrm{O}$ & C-O stretching [30] & \\
\hline 11000 & 909 & Ring & Ring stretching [30] & \\
\hline 13333 & 750 & $\mathrm{~N}-\mathrm{H}$ & N-H out-of-plane bending [29-30] & Amide V \\
\hline $\begin{array}{l}14390 \\
15504 \\
\end{array}$ & $\begin{array}{l}695 \\
645 \\
\end{array}$ & & $\begin{array}{l}\mathrm{O}-\mathrm{C}-\mathrm{N} \text { bending }[29] \text { and/or } \mathrm{O}-\mathrm{H} \text { out-of-plane bending [30] } \\
\text { as above }\end{array}$ & $\begin{array}{l}\text { Amide IV } \\
\text { as above }\end{array}$ \\
\hline 16950 & 590 & $\mathrm{C}=\mathrm{O}$ & $\mathrm{C}=\mathrm{O}$ out - of-plane bending [29] & Amide VI \\
\hline
\end{tabular}




\section{References}

1. R. Hooke, Micrographia: or Some Physiological Descriptions of Minute Bodies Made by Magnifying Glasses with Observations and Inquiries Thereupon (J. Martyn and J. Allestry, 1665).

2. D. H. Goldstein,"Polarization properties of Scarabaeidae," Appl.Opt. 45, 7944-7950 (2006).

3. H. Arwin, "Application of ellipsometry techniques to biological materials," Thin Solid Films 519, 2589-2592 (2011).

4. S. Berthier, E. Charron, and J. Boulenguez, "Morphological structure and optical properties of the wings of Morphidae," Insect Sci. 13, $145-157$ (2006).

5. B. Bhushan, "Biomimetics: lessons from nature-an overview," Phil. Trans. R. Soc. A 367, 1445-1486 (2009).

6. Y. Bar-Cohen, Biomimetics Biologically Inspired Technologies (Taylor \& Francis Group, 2006).

7. Y. Chen, J. Gu, S. Zhu, T. Fan, D. Zhang, and Q. Guo, "Iridescent large-area $\mathrm{ZrO}_{2}$ photonic crystals using butterfly as templates,"Appl. Phys. Lett. 94, 053901 (2009).

8. P. Vukusic, R. Kelly, and I. Hooper, "A biological sub-micron thickness optical broadband reflector characterized using both light and microwaves," J. R. Soc. Interface 6, S193-S201 (2009).

9. K. E. Andersson and C. Åkerlind, "A review of materials for spectral design coatings in signature management applications," Proc. SPIE 84 92530Y, 1-20 (2014).

10. W. L. Allen, I. C. Cuthill, N. E. Scott-Samuel, and R. Baddeley, "Why the leopard got its spots: relating pattern development to ecology in felids," Proc. R. Soc. B. 1-8 (2010).

11. G. S. Watson, and J. A. Blach, "Characterisation of cuticular nanostructures on surfaces of insects by atomic force microscopy- 'mining' evolution for smart structures," Proc. SPIE 4934, 378-385 (2002).

12. P. R. Stoddart, P. J. Cadusch, T. M. Boyce, R. M. Erasmus, and J. D. Comins, "Optical properties of chitin: surface-enhanced Raman scattering substrates based on antireflection structures on cicada wings," Nanotechnology 17, 680-686 (2006).

13. P. Vukusic, B. Hallam, and J. Noyes, "Brilliant whiteness in ultrathin beetle scales," Science 315, 348 (2007).

14. S. M. Luke, B. T. Hallam, and P. Vukusic, "Structural optimization for broadband scattering in several ultra-thin white beetle scales," Appl. Opt. 49, 4246-4254 (2010).

15. M. Burresi, L. Cortese, L. Pattelli, M. Kolle, P. Vukusic, D. S. Wiersma, U. Steiner, and S. Vignolini, "Bright-white beetle scales optimise multiple scattering of light”, Scientific Reports 4, 6075 (2014).

16. B. T. Hallam, A. G. Hiorns, and P. Vukusic, "Developing optical efficiency through optimized coating structure: biomimetic inspiration from white beetles," Appl. Opt. 48, 3243-3249 (2009).

17. J. Yip, S.-P. Ng, and K.-H. Wong, "Brilliant whiteness surfaces from electrospun nanofiber webs," Text. Res. J. 79, 771-779 (2009).

18. H. Arwin, T. Berlind, B. Johs, and K. Järrendahl, "Cuticle structure of scarab beetle Cetonia aurata analyzed by regression analysis of Mueller-matrix ellipsometric data," Opt. Expr. 21, 22645-22656 (2013).

19. I. Hodgkinson, S. Lowrey, L. Bourke, A. Parker, and M. W. McCall, "Mueller-matrix characterization of beetle cuticle: polarized and unpolarized reflections from representative architectures," Appl. Opt. 49, 4558-4567 (2010).

20. H. Arwin, R. Magnusson, J. Landin, and K. Järrendahl, "Chirality-induced polarization effects in the cuticle of scarab beetles: 100 years after Michelson,” Phil. Mag. 92 1583-1599 (2012).

21. H. Arwin, R. Magnusson, L. Fernández del Río, C. Åkerlind, E. Muñoz-Pineda, J. Landin, A. Mendoza-Galván, and K. Järrendahl. Materials Today: Proceedings 1S 155-160, (2014).

22. CIE Technical Report, Colorimetry, 3d ed. draft CIE 15.3:2004, http://cie.mogi.bme.hu/cie arch/kee/div1/tc148.pdf .

23. J. M. Bennett, and L. Mattson, Introduction to Surface Roughness and Scattering, second edition (Optical Society of America, 1999).

24. J. Jafolla, D. Thomas, J. Hilgers, B. Reynolds, and C. Blasband, "Theory and measurement of bidirectional reflectance for signature analysis," Proc. SPIE 3699, 2-15 (1999).

25. J. C. Stover, Optical Scattering: Measurement and Analysis (McGraw-Hill, Inc., 1990).

26. R. M. A. Azzam, and N. M. Bashara, Ellipsometry and Polarized Light (North Holland, 1987).

27. J. Lafait, C. Andraud, S. Berthier, J. Boulenguez, P. Callet, S. Dumazet, M. Rassart, and J.-P. Vigneron, "Modeling the vivid white color of the beetle Calothyrza margaritifera," Mater. Sci. Eng. B 169, 16-22 (2010).

28. N. Pauler, Paper Optics (AB Lorentzen \& Wettre, 2002).

29. J. Kong, and S. Yu, "Fourier transform infrared spectroscopic analysis of protein secondary structures," Acta Biochim. Biophys. Sin. 39, 549-559 (2007).

30. C. Palpandi, V. Shanmugam, and A. Shanmugam, "Extraction of chitin and chitosan from shell and operculum of mangrove gastropod Nerita (Dostia) crepidularia Lamarck," Int. J. Med. Med. Sci. 1, 198-205 (2009).

31. C. Mobley (principal author), Ocean optics web book, http://www.oceanopticsbook.info/view/surfaces/lambertian_brdfs (January 26th, 2015)

32. D. E. Azofeifa, H. J. Arguedas, and W. E. Vargas,”Optical properties of chitin and chitosan biopolymers with application to structural color analysis," Optical materials 35, 175-183 (2012).

33. A. Mendoza-Galván, E. Muñoz-Pineda, K. Järrendahl, and H. Arwin, "Evidence for a dispersion relation of optical modes in the cuticle of the scarab beetle Cotinis mutabilis," Opt. Mat. 4, 2484-2496 (2014).

34. D. A. Burns and E. W. Ciurczak (Eds.) "Handbook of Near-Infrared Analysis," 13, in Practical Spectroscopy Series, (Marcel Dekker, Inc., 1992).

35. Infrared Spectroscopy: Its Use in the Coatings Industry, by Infrared Spectroscopy Committee of the Chicago Society for Paint Technology, (Federation of Societies for Paint Technology, 1969). 